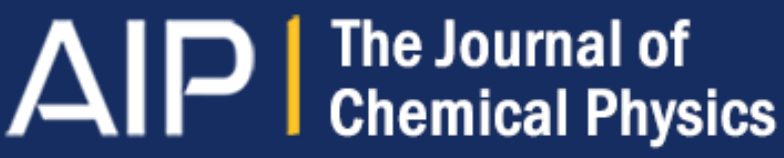

\section{The bridge functions of molten salts}

Çetin Taşseven, Luis Enrique González, Moises Silbert, Olga Alcaraz, and Joaquim Trullàs

Citation: The Journal of Chemical Physics 115, 4676 (2001); doi: 10.1063/1.1392366

View online: http://dx.doi.org/10.1063/1.1392366

View Table of Contents: http://scitation.aip.org/content/aip/journal/jcp/115/10?ver=pdfcov

Published by the AIP Publishing

\section{Articles you may be interested in}

First principles molecular dynamics of molten Nal: Structure, self-diffusion, polarization effects, and charge transfer

J. Chem. Phys. 127, 094506 (2007); 10.1063/1.2768968

First principles molecular dynamics of molten $\mathrm{NaCl}$

J. Chem. Phys. 126, 124502 (2007); 10.1063/1.2711187

A polarizable ion model for the structure of molten Agl

J. Chem. Phys. 126, 021105 (2007); 10.1063/1.2432346

Density-functional-based molecular-dynamics simulations of molten salts

J. Chem. Phys. 123, 134510 (2005); 10.1063/1.2038888

Molecular dynamics simulation of molten sodium chlorate

J. Chem. Phys. 119, 8002 (2003); 10.1063/1.1609983

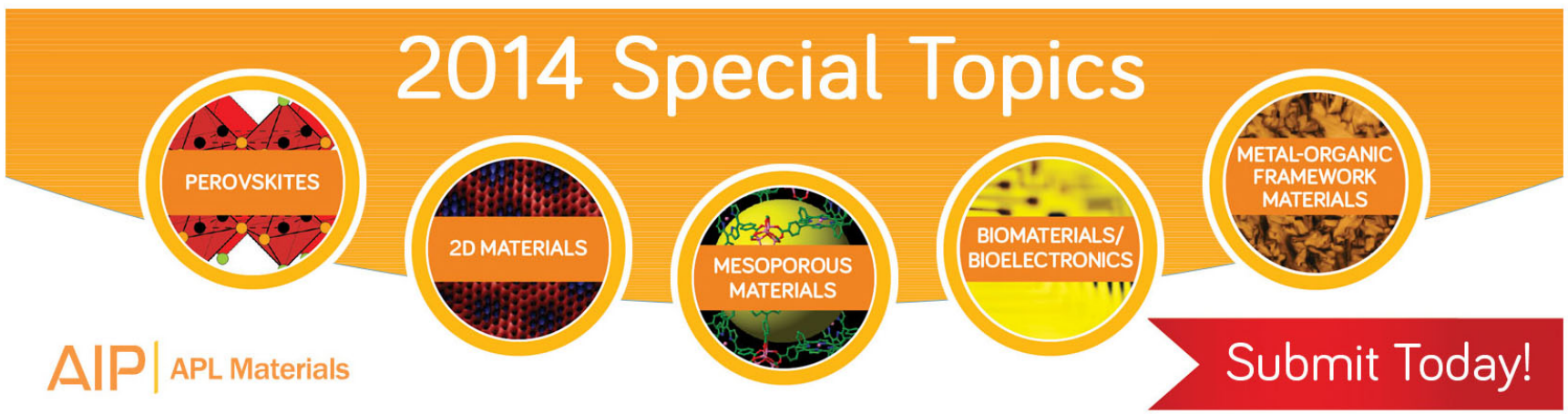




\title{
The bridge functions of molten salts
}

\author{
Çetin Taşseven \\ Department of Physics, Faculty of Science and Art, Yildiz Technical University, Şişli 80270, Istanbul, Turkey \\ Luis Enrique González and Moises Silbert ${ }^{\text {a) }}$ \\ Departamento de Física Teórica, Facultad de Ciencias, Universidad de Valladolid, 47011 Valladolid, Spain
}

Olga Alcaraz and Joaquim Trullàs

Departament de Física i Enginyeria Nuclear, Universitat Politènica de Catalunya, Campus Nord, Mòdul B4, 08034 Barcelona, Spain

(Received 16 November 2000; accepted 20 June 2001)

\begin{abstract}
The bridge functions of molten $\mathrm{NaCl}$ and $\mathrm{AgI}$ near melting were obtained by using the model potentials of Born-Huggins-Mayer for $\mathrm{NaCl}$ and Vashishta-Rahman for AgI. The calculations of the bridge functions involved molecular dynamics simulations, the extension of the procedure originally proposed by Poll et al. [Phys. Rev. A 37, 1672 (1988)] and the numerical solution of the Ornstein-Zernike equations. The calculated bridge functions do not conform with the universality ansatz. They also differ from the bridge functions obtained for model electrolytes. Following the results obtained for electrolytes and those of this work for molten salts, it is conjectured that the universality ansatz for the bridge functions does not apply for systems whose attractive interactions play a decisive role in their structural ordering. (C) 2001 American Institute of Physics.
\end{abstract}

[DOI: $10.1063 / 1.1392366$ ]

\section{INTRODUCTION}

This work is concerned with studies of the bridge functions for two classes of model molten salts, as obtained from computer simulations.

The bridge function $B(r)$ may be obtained from the theory of liquids, for a given pair potential $\phi(r)$, if the pair distribution function $g(r)$ is known for all values of $r$. Alas, this knowledge is not accessible to us either from experiments or from computer simulations. In the former our knowledge of $g(r)$ or, more precisely, the structure factor $S(q)$ is limited to the range of angles that a particular neutron or x-ray diffractometer can cover. Computer simulations are limited by the size of the simulation box, namely the number of particles used in the simulations.

In order to overcome these difficulties, and thus make possible the formulation of thermodynamic self-consistent theories of liquids, Lado, ${ }^{1}$ and later Rosenfeld and Ashcroft, ${ }^{2}$ proposed approximating the bridge function, with the bridge function of a suitably chosen reference system at the appropriate thermodynamic state. Moreover, the latter put forward the ansatz of the universality of the bridge function, namely that the main features of the bridge function are largely independent of the chosen reference system.

The preceding approaches took on various acronyms, such as RHNC, ${ }^{1}$ MHNC, ${ }^{2}$ VMHNC, ${ }^{3}$ or variations thereof. These were extended to multicomponent systems by Enciso et $a l^{4}{ }^{4}$ and González et al. ${ }^{5}$

More recently Rosenfeld $^{6,7}$ has introduced a selfconsistent fundamental measure free-energy density func-

\footnotetext{
a) Author to whom correspondence should be addressed. On study leave from the School of Chemical Sciences, University of East Anglia, Norwich NR4 7TJ, UK. Electronic mail: m.silbert@uea.ac.uk
}

tional that provides simple expressions for the bridge functional. Again he postulates the universality ansatz for the bridge functional, which is approximated by that for hard spheres. This approach has led to highly accurate results for a variety of liquids and liquid mixtures, as well as for inhomogeneous liquids. ${ }^{8}$

Most of the preceding studies neglected to address the case of ionic melts. For the purposes of this work, ionic melts are restricted to classical systems where the Coulomb interaction is explicitly present in the potential energy of interaction, and involves the interaction of both like and unlike ions. Such a definition excludes the important case of classical plasmas, where the interaction between the ions is purely repulsive. It also excludes liquid metals and chargestabilized colloids where the effective interionic potential is screened by free electrons in the first case, and by counterions/added salt in the other. In all of these cases, where the structure is basically decided by the repulsive interaction between the particules that make up the system, the universality ansatz works very well.

In the class of ionic melts we are concerned with, the long-range attractive interaction between unlike ions plays a decisive role in the structural ordering of these systems. Most of the calculations involving bridge functions in this class of melts have been, broadly, of two types. One in which the bridge functions are assumed to satisfy the universality ansatz; ${ }^{9,10}$ the other includes explicitly the contribution of the long range Coulomb potential to the bridge functions. ${ }^{11-16}$ The second approach was largely motivated by the shortcomings of the $\mathrm{HNC}$ approximation in accounting for the properties of electrolytes, specifically $2-2$ aqueous atomic electrolytes at low molar concentrations. The bridge functions were introduced either by calculating directly the first, or 
first two, terms of the bridge function graph expansion, ${ }^{11,12}$ or by proposing approximate schemes for the bridge functions, ${ }^{13-15}$ which resulted in the so-called IPY, INV, and IPY2 closures. In those cases where the bridge functions were either tabulated ${ }^{12}$ or shown as figures, ${ }^{13,14}$ they exhibit similar qualitative behavior. The bridge functions for the like charges is positive, $B_{++}(r)=B_{--}(r)$ in the examples discussed in all these papers, and tend almost monotonically to zero, except for a discontinuity at $r=2 \sigma$, the distance at which the principal maximum is observed in the like pair distribution functions. $B_{ \pm}(r)$ is negative and also decays almost monotonically to zero. The Monte Carlo (MC) simulations of Bresme et al. ${ }^{16}$ confirm these trends, and also find that the INV approximation works extremely well at normal and high temperatures. The main message of these results is that there is no evidence to support the universality ansatz of the bridge functions in model electrolytes.

The main aim of this article is, precisely, to carry out for model molten salts studies similar to those by Bresme et al. ${ }^{16}$ for model electrolytes. Two of the papers that, to our knowledge, are concerned with the molten salts regime, ${ }^{9,10}$ assume the validity of the universality ansatz. These authors find that their calculated partial structures are in better agreement, vis à vis computer simulations, by using the universality ansatz. However, the question raised in this work is whether the bridge functions derived from simulations of model molten salts conform with the universality ansatz for the bridge functions. We shall show in this work that, like electrolytes, the bridge functions of molten salts do not conform with the universality ansatz. Actually, Babu and Ichiye ${ }^{15}$ did also consider the molten salts regime, and their Fig. 4 compares the calculated IPY2 and HNC pair distribution functions with Monte Carlo (MC) results for charged spheres with equal diameters. The difference between the MC and $\mathrm{HNC} g_{\alpha \beta}(r)$ do give an inkling of the behavior of the bridge functions which, unfortunately, are not shown.

In order to obtain $B_{\alpha \beta}(r)$ from computer simulations, specifically molecular dynamics (MD) simulations, it is necessary to develop a reliable procedure that allows for the extension of the MD results beyond the range of the MD simulation box. This is another aim of this work. We have extended to the case of molten salts the procedure originally proposed by Poll et al. ${ }^{17}$ to extend simulation data obtained for the classical one-component plasma (OCP). Unlike OCP, where the potential is always repulsive, in molten salts we have to take care of both repulsive and attractive interactions.

We obtained $B_{\alpha \beta}(r)$ for two classes of model molten salts near their melting points. The first is concerned with molten $\mathrm{NaCl}$, regarded as the archetypal "simple" molten salt, using the Born-Huggins-Mayer potentials as parametrized by Fumi and Tosi. ${ }^{18}$ The second is concerned with molten AgI, regarded as the archetypal "superionic" melt, using the potentials of Vashishta and Rahman. ${ }^{19}$

The layout of the article is as follows. In Sec. II we go briefly through the necessary theory and describe the extension of the Poll et al. procedure. In Sec. III we present the results of our calculations. Finally, in Sec. IV, we sum up and discuss our results.
TABLE I. Parameters of the Born-Huggins-Mayer potentials for molten $\mathrm{NaCl}$ (see Ref. 21).

\begin{tabular}{cccc}
\hline \hline & $b_{\alpha \beta}(\mathrm{eV})$ & $C_{\alpha \beta}\left(\mathrm{eV} \AA^{6}\right)$ & $D_{\alpha \beta}\left(\mathrm{eV} \AA^{8}\right)$ \\
\hline++ & 0.264 & 1.05 & 0.499 \\
+- & 0.211 & 6.99 & 8.68 \\
-- & 0.158 & 72.4 & 145.5 \\
\hline \hline
\end{tabular}

\section{BRIDGE FUNCTIONS. METHOD OF CALCULATION}

\section{A. Integral equations}

For an ionic liquid mixture of $\nu$ species at temperature $T$ and density $\rho$, the direct correlation functions $c_{\alpha \beta}(r)$ between species $\alpha$ and $\beta$, separated by a distance $r=\mid \mathbf{r}_{\alpha}$ $-\mathbf{r}_{\beta} \mid$, is defined by the Ornstein-Zernike $(\mathrm{OZ})$ relations,

$$
h_{\alpha \beta}(r)=c_{\alpha \beta}(r)+\rho \sum_{\gamma=1}^{\nu} x_{\gamma} \int d \mathbf{r}^{\prime} c_{\alpha \gamma}\left(r^{\prime}\right) h_{\gamma \beta}\left(\left|\mathbf{r}-\mathbf{r}^{\prime}\right|\right),
$$

where the total correlation function $h_{\alpha \beta}(r)=g_{\alpha \beta}(r)-1$, and $x_{\alpha}$ is the concentration of particles of species $\alpha$, with charge $Z_{\alpha}$, such that $\sum_{\alpha=1}^{\nu} x_{\alpha}=1$ and subject to the overall charge neutrality condition $\Sigma_{\alpha=1}^{\nu} x_{\alpha} Z_{\alpha}=0$.

The bridge functions follow from the exact closure relation, ${ }^{20}$

$$
B_{\alpha \beta}(r)=-\beta \phi_{\alpha \beta}(r)-\ln g_{\alpha \beta}(r)+g_{\alpha \beta}(r)-1-c_{\alpha \beta}(r),
$$

where $\beta \equiv\left(k_{B} T\right)^{-1}$, and $k_{B}$ is the Boltzmann constant. We note that Eq. (2) is the exact closure for the pair correlation functions even in the presence of many body forces. We follow Rosenfeld and Ashcroft ${ }^{2}$ in writing the bridge functions as $B_{\alpha \beta}(r)=-E_{\alpha \beta}(r)$, where $E_{\alpha \beta}(r)$ denote the elementary graphs functions defined in Ref. 20. Note that other authors (cf. Refs. 10-16) follow Lado ${ }^{1}$ in identifying the bridge functions as the elementary graphs functions, i.e., $B_{\alpha \beta}(r)=E_{\alpha \beta}(r)$.

In this work we shall only be concerned with effective pair potentials. The effective pair potentials for molten $\mathrm{NaCl}$ are given by ${ }^{18}$

$$
\begin{aligned}
\phi_{\alpha \beta}^{\mathrm{NaCl}}(r)= & b_{\alpha \beta} \exp \left[\gamma\left(\sigma_{\alpha}+\sigma_{\beta}-r\right)\right] \\
& +\frac{Z_{\alpha} Z_{\beta} e^{2}}{r}+\frac{C_{\alpha \beta}}{r^{6}}-\frac{D_{\alpha \beta}}{r^{8}},
\end{aligned}
$$

where $\sigma_{\alpha}$ and $\sigma_{\beta}$ denote the ionic radii, and $b_{\alpha \beta}, \gamma, C_{\alpha \beta}$, and $D_{\alpha \beta}$ are parameters whose meaning is discussed in Ref. 18 (see also Ref. 21). In this work we assume $Z=Z_{+}$ $=\left|Z_{-}\right|=1, \quad \gamma=3.155 \AA^{-1}, \quad \sigma_{+}=1.170 \AA, \quad$ and $\quad \sigma_{-}$ $=1.585 \AA$. The values of $b_{\alpha \beta}, C_{\alpha \beta}$, and $D_{\alpha \beta}$ are given in Table I. ${ }^{21}$ form $^{19}$

For molten AgI the effective pair potentials are of the

$$
\phi_{\alpha \beta}^{\mathrm{AgI}}(r)=\frac{H_{\alpha \beta}}{r^{\eta_{\alpha \beta}}}+\frac{Z_{\alpha} Z_{\beta} e^{2}}{r}+\frac{P_{\alpha \beta}}{r^{4}}+\frac{C_{\alpha \beta}}{r^{6}},
$$

where $\eta_{\alpha \beta}, H_{\alpha \beta}, P_{\alpha \beta}$, and $C_{\alpha \beta}$ are parameters whose meaning is discussed in Ref. 19 (see also Ref. 22). In this 
TABLE II. Parameters of the Vashishta and Rahman potentials for molten AgI (see Ref. 19).

\begin{tabular}{ccccc}
\hline \hline & $\eta_{\alpha \beta}$ & $H_{\alpha \beta}\left(\mathrm{eV} \AA^{\eta_{\alpha \beta}}\right)$ & $P_{\alpha \beta}\left(\mathrm{eV} \AA^{4}\right)$ & $C_{\alpha \beta}\left(\mathrm{eV}^{6}\right)$ \\
\hline++ & 11 & 0.2132 & 0 & 0 \\
+- & 9 & 1548.5 & 16.9 & 0 \\
-- & 7 & 6431.5 & 33.8 & 99.8 \\
\hline \hline
\end{tabular}

work we assume $Z=Z_{+}=\left|Z_{-}\right|=0.6$. The values of the parameters $\eta_{\alpha \beta}, H_{\alpha \beta}, P_{\alpha \beta}$, and $C_{\alpha \beta}$ are given in Table II. ${ }^{22}$

The OZ relations, together with the closure relations, were solved numerically using the method originally proposed by Gillan. ${ }^{23}$ Accuracy in the numerical solutions requires that, in the process of Fourier transforming Eq. (1), we first substract the Coulomb interaction contribution to the correlation functions, and later add its analytical Fourier transform directly. The numerical solution of Eq. (1) involves two previous stages; first, obtaining results from the MD simulations (see Sec. III), and second, extending the MD data beyond the simulation box. We discuss below the procedure used for the latter.

\section{B. The extension method}

The $g_{\alpha \beta}\left(r \leqslant R_{e}<L / 2\right)$ from MD simulations are extended to $r \geqslant R_{e}$, taking care of the discontinuity at the extension point $R_{e}$. We have adapted and generalized the method proposed by Poll et al. ${ }^{17}$ for the OCP to molten salts, so that

$$
\begin{aligned}
& g_{\alpha \beta}(r)=g_{\alpha \beta}^{\mathrm{MD}}(r), \quad r \leqslant R_{e}, \\
& c_{\alpha \beta}(r)=-\beta \phi_{\alpha \beta}(r)+A_{\alpha \beta} \frac{\exp \left(-\lambda_{\alpha \beta} r\right)}{r}, \quad r>R_{e} .
\end{aligned}
$$

The discontinuities in the extended $g_{\alpha \beta}(r)$ and $c_{\alpha \beta}(r)$ are due to the fact we are using exact relations for $r \leqslant R_{e}$ but approximate relations for $r>R_{e}$. The first term on the right hand side of $c_{\alpha \beta}(r)$ ensures its correct asymptotic behavior; the second term is used to correct any discontinuity in $c_{\alpha \beta}(r)$. The parameters $A_{\alpha \beta}$ and $\lambda_{\alpha \beta}$ are chosen to enforce continuity in the function and its derivative. We have also replaced $-\beta \phi_{\alpha \beta}(r)$ in Eq. (5) for $-\beta \phi_{\alpha \beta}(r)+g_{\alpha \beta}^{\mathrm{HNC}}(r)$ $-1-\ln g_{\alpha \beta}^{\mathrm{HNC}}(r)$, where the superscript HNC denotes the hypernetted chain closure, but found no differences in the results for the bridge functions. The results shown in this work are those obtained using Eq. (5). We note that, unlike Poll et al., we are not splitting $c_{\alpha \beta}(r)$ into their short- and long-range contributions in order to make the continuous matching of the functions; it is not necessary within the procedure used in this work. The decomposition, as stated in the preceding subsection, only enters as a standard procedure in the Gillan algorithm ${ }^{23}$ used to solve numerically the $\mathrm{OZ}$ equations.

\section{RESULTS}

In the $\mathrm{MD}$ simulations for $\mathrm{NaCl}$ and $\mathrm{AgI}$ we consider $N=216$ ions (N/2 anions and $N / 2$ cations) placed in a cubic box of side $L$, with periodic boundary conditions at temperature $T$ and ionic density $\rho$. We have used the algorithm of
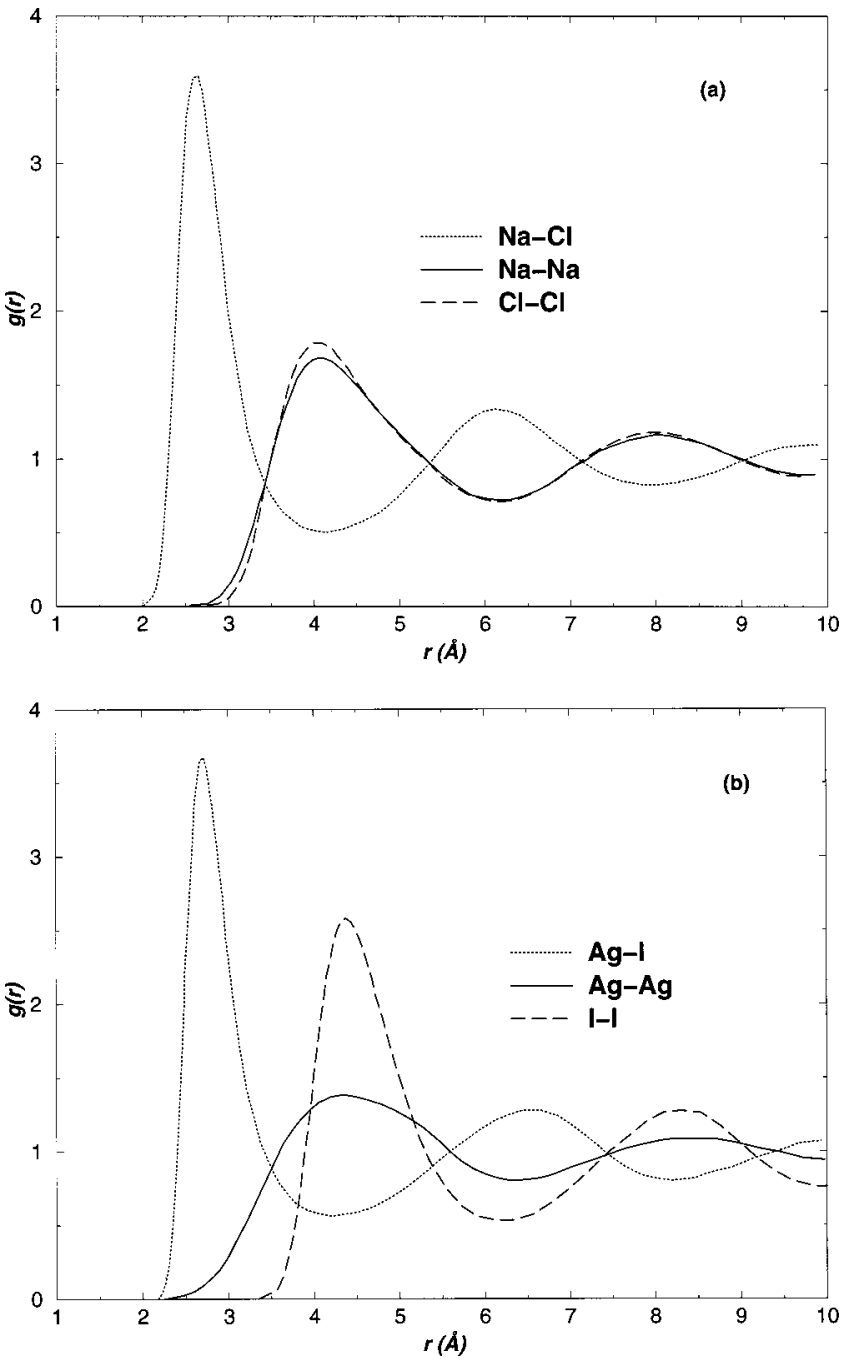

FIG. 1. Extended pair distribution functions for molten $\mathrm{NaCl}$ and $\mathrm{AgI}$. $g_{++}:$solid line; $g_{--}$: dashed line; $g_{+-}$: dotted line. (a) Molten $\mathrm{NaCl}$ at $T=1165 \mathrm{~K}$ and $\rho=0.0314$ ions $/ \AA^{3}$. (b) Molten $\mathrm{AgI}$ at $T=933 \mathrm{~K}$ and $\rho$ $=0.0281$ ions $/ \AA^{3}$.

Beeman ${ }^{24}$ with a time step $\Delta t=5 \times 10^{-15} \mathrm{~s}$, and the Ewald method $^{21}$ to account for the long-range Coulomb interactions.

For molten $\mathrm{NaCl}$ the input temperature and ionic density are $T=1165 \mathrm{~K}$ and $\rho=0.0314$ ions $/ \AA^{3}$, whereas for molten AgI they are $T=933 \mathrm{~K}$ and $\rho=0.0281$ ions $/ \AA^{3}$, respectively.

The extension point $R_{e}$ is taken to be $6.5 \AA$, which is less than $L / 2$ for both simulated systems.

The extended $g_{\alpha \beta}(r)$ is used to solve iteratively the OZ equations (1) until the convergence criteria,

$$
\left[\sum_{i}\left|c_{i}(r)-c_{i+1}(r)\right|^{2} r^{2}\right]^{1 / 2}<10^{-5}
$$

was achieved for each of the direct correlation functions $c_{\alpha \beta}(r)(\alpha, \beta=+,-)$. This ensures consistency between the $g_{\alpha \beta}^{\mathrm{MD}}(r)$ data and the solution of the OZ equations. The extended pair distribution functions, which obviously coincide with the MD ones for distances shorter than $R_{e}$, are shown in Fig. 1. As another check of our results we have compared the total structure factors $S(k)$ of molten $\mathrm{NaCl}$ and $\mathrm{AgI}$ cal- 

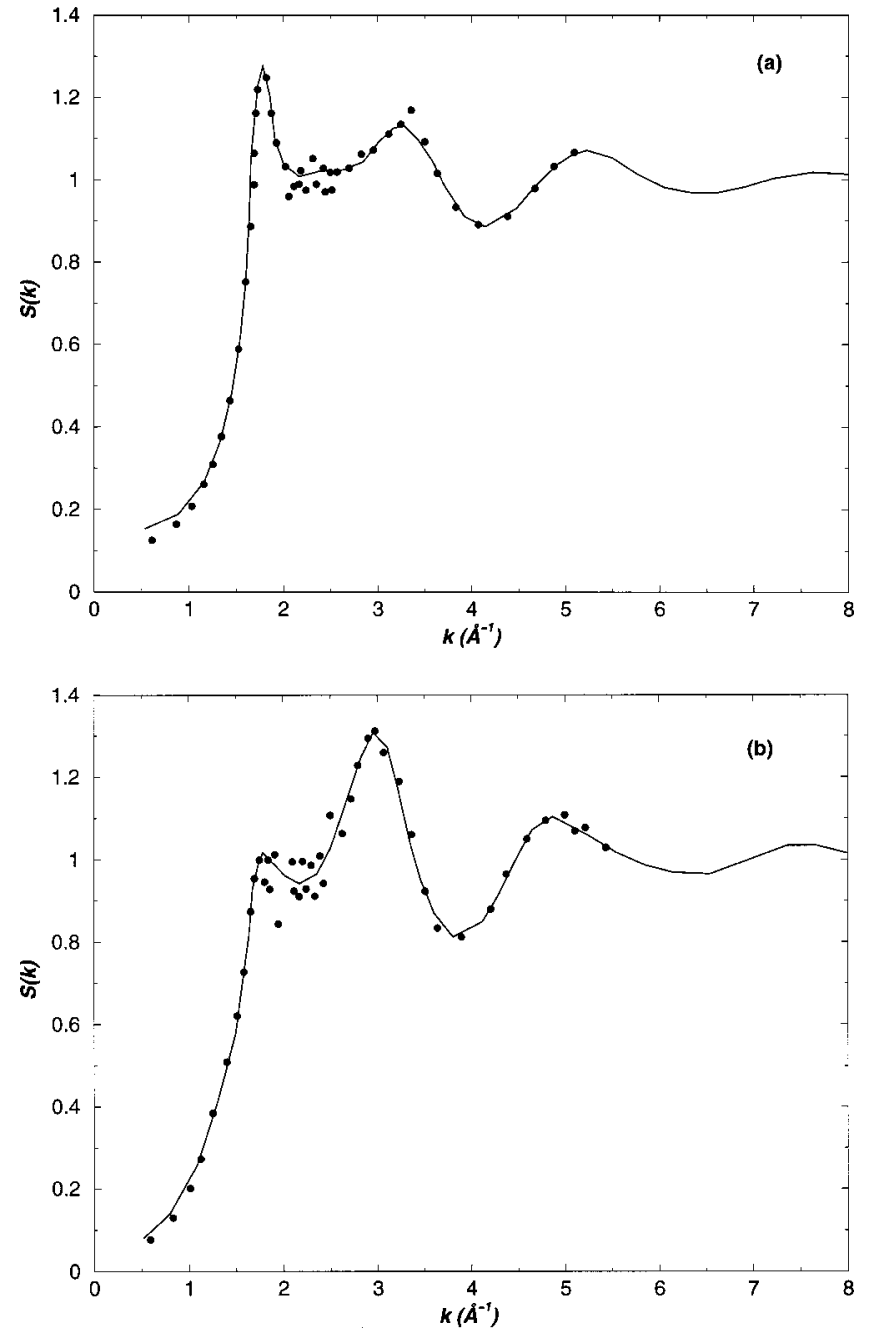

FIG. 2. Total structure factor for molten $\mathrm{NaCl}$ and AgI. Solid line: results obtained from Fourier transformation of the extended pair distribution functions. Full circles: results obtained directly from the MD simulation. (a) Molten NaCl. (b) Molten AgI.

culated directly from the simulations and those obtained by the Fourier transform of the extended $g_{\alpha \beta}(r)$. The agreement between the two is very good, within the estimated $2 \%$ error bar of the simulations (see Fig. 2).

With the extended $g_{\alpha \beta}(r)$ and $c_{\alpha \beta}(r)$, and the given potentials, we have calculated the bridge functions $B_{\alpha \beta}(r)$. Figure 3(a) shows the $B_{\alpha \beta}(r)$ corresponding of molten $\mathrm{NaCl}$, while Fig. 3(b) shows those for molten AgI. The first point to notice is that, in spite of the differences in the potentials, both sets of $B_{\alpha \beta}(r)$ are qualitatively very similar. In both cases the bridge functions for the like ions take negative values in their first minimum. Also, both exhibit a principal peak in $B_{ \pm}(r)$ at around 2.2-2.3 $\AA$. These features are not present in Rosenfeld's universal hard-sphere bridge functions, where $B_{\alpha \beta}(r) \geqslant 0$ for all $r$. Our results are also qualitatively different from those obtained by Bresme et al. ${ }^{16}$ in their MC simulations of the primitive electrolyte model. Note that in their work $B_{\alpha \beta}(r)=E_{\alpha \beta}$, so that there is a difference in sign between their bridge functions and ours.
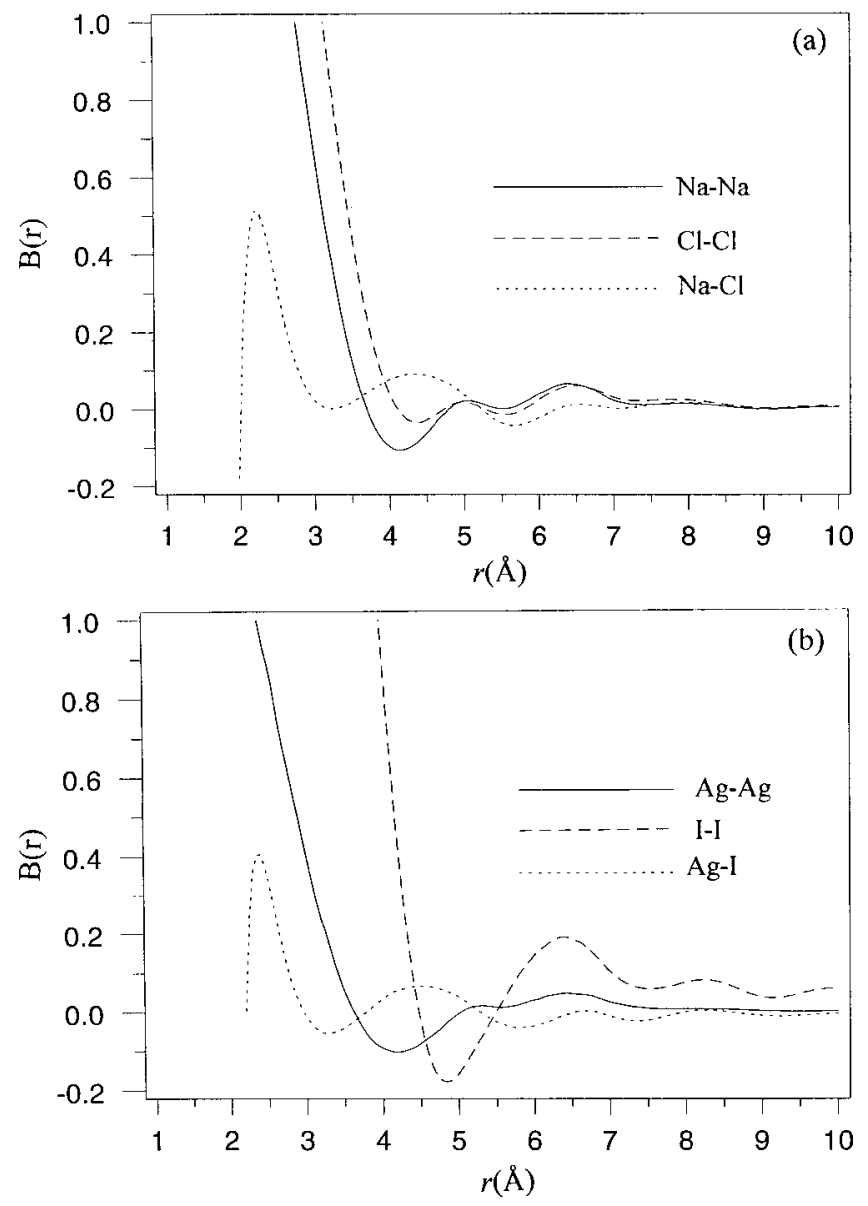

FIG. 3. Bridge functions for molten $\mathrm{NaCl}$ and AgI. $B_{++}:$solid line; $B_{--}$: dashed line; $B_{+-}$: dotted line. (a) Molten NaCl. (b) Molten AgI.

\section{DISCUSSION}

We have used MD simulation data, the extension procedure originally proposed by Poll et al. and the OZ equations, to obtain the bridge functions for two model molten salts, $\mathrm{NaCl}$ and $\mathrm{AgI}$, near melting. We have not checked the sensitivity of the extension procedure; whence it is likely that the final form of the bridge functions presented in this work do depend in some way on the underlying assumptions made when writing down Eq. (5). ${ }^{17}$ Moreover, the relatively small number of particles used in the simulations, while perfectly reasonable for the calculations of $g_{\alpha \beta}(r)$, do introduce important error bars in the final calculation of the bridge functions. Besides the error bars inherent in the simulations, there are also propagating errors arising from the calculation of the direct correlation functions through the iterative procedure that employs the extension method and the OZ equations.

Notwithstanding the preceding caveats we submit that the bridge functions presented in Figs. 1(a) and 1(b) are qualitatively correct within the range presented in the figures. We find that the $B_{\alpha \beta}(r)$ obtained using model potentials that generate the correct structure of molten $\mathrm{NaCl}$ and $\mathrm{AgI}$ near melting are qualitatively similar. However, they differ from Rosenfeld's universal hard-sphere bridge functions as well as those obtained for model electrolytes. As a result of these findings, it is unlikely that approximations, such as INV, that predict very good bridge functions for model electrolytes, 
within a temperature range, will be good approximations in the molten salts regime. It is possible that the IPY2 and related approximations may predict bridge functions that are in reasonably good qualitative agreement with those obtained in this work, besides improving the pair distribution functions. This is a point worth investigating, as the IPY2 may provide almost analytic expressions for the $B_{\alpha \beta}(r)$.

Both the results for electrolytes and molten salts fail to conform with the universality ansatz. In these classes of systems the long-range attractive forces introduce a degree of ordering that affects their structure in a significant way. Whence, we suggest that the universality ansatz is only relevant for those systems where the structure is mainly determined by the repulsive forces. If our conjecture is correct, then another important class of liquids, namely polar liquids, will not satisfy either the universality ansatz. Recent work (cf. the recent review by Teixeira et al. ${ }^{25}$ ) has shown that the ordering due to polar interactions affect in a decisive way their structure, so much so that hard-sphere dipoles are unable to form a liquid. It is also unlikely that the bridge functional, derived from fundamental measure free-energy density functional introduced by Rosenfeld, ${ }^{6,8}$ is capable of accounting for the bridge functions in liquid electrolytes or molten salts. The main reason is that the free-energy is geometrically based, actually inspired by the ideas of the scale particle theory. ${ }^{26}$ Such a construct largely ignores the contributions from the attractive forces to the liquid structure.

\section{ACKNOWLEDGMENTS}

The authors thank David González for useful discussions. This work was partially supported by the Junta de Castilla y León of Spain (VA 70/99), CICYT of Spain
(PB98-0368), and NATO (CRG971173). One of the authors (M.S.) gratefully acknowledges the financial support of the Ministry of Education and Science of Spain.

${ }^{1}$ F. Lado, Phys. Rev. A 8, 2548 (1973).

${ }^{2}$ Y. Rosenfeld and N. W. Ashcroft, Phys. Rev. A 20, 1208 (1979).

${ }^{3}$ Y. Rosenfeld, J. Stat. Phys. 42, 437 (1986).

${ }^{4}$ E. Enciso, F. Lado, M. Lombardero, J. L. F. Abascal, and S. Lago, J. Chem. Phys. 87, 2249 (1987).

${ }^{5}$ L. E. Gonzalez, D. J. Gonzalez, and M. Silbert, Phys. Rev. A 45, 3803 (1992).

${ }^{6}$ Y. Rosenfeld, Phys. Rev. Lett. 63, 980 (1989).

${ }^{7}$ Y. Rosenfeld, J. Chem. Phys. 93, 4305 (1990).

${ }^{8}$ See Y. Rosenfeld, J. Phys.: Condens. Matter 8, 9289 (1996), and references therein.

${ }^{9}$ C. Caccamo, G. Malescio, and L. Reatto, J. Chem. Phys. 81, 4093 (1984).

${ }^{10}$ A. I. Livshits and G. A. Martynov, J. Chem. Phys. 90, 6603 (1989).

${ }^{11}$ P. J. Rossky, J. B. Dudowicz, B. L. Tembe, and H. L. Friedman, J. Chem. Phys. 73, 3372 (1980)

${ }^{12}$ R. Bacquet and P. J. Rossky, J. Chem. Phys. 79, 1419 (1983).

${ }^{13}$ T. Ichiye and A. D. J. Haymet, J. Chem. Phys. 93, 8954 (1990).

${ }^{14}$ D.-M. Duh and A. D. J. Haymet, J. Chem. Phys. 97, 7716 (1992).

${ }^{15}$ C. S. Babu and T. Ichiye, J. Chem. Phys. 100, 9147 (1994).

${ }^{16}$ P. Bresme, E. Lomba, J. J. Weis, and J. L. F. Abascal, Phys. Rev. E 51, 289 (1995).

${ }^{17}$ P. D. Poll, N. W. Ashcroft, and H. E. de Witt, Phys. Rev. A 37, 1672 (1988).

${ }^{18}$ F. G. Fumi and M. P. Tosi, J. Phys. Chem. Solid State 25, 31 (1964).

${ }^{19}$ P. Vashishta and A. Rahman, Phys. Rev. Lett. 40, 1337 (1978).

${ }^{20}$ G. Stell, in The Equilibrium Theory of Classical Fluids, edited by H. L. Frisch and J. L. Lebowitz (Benjamin, New York, 1964), p. II-71.

${ }^{21}$ M. J. L. Sangster and M. Dixon, Adv. Phys. 25, 247 (1976).

${ }^{22}$ A. J. Stafford, M. Silbert, J. Trullàs, and A. Giró, J. Phys.: Condens. Matter 2, 6631 (1990).

${ }^{23}$ M. J. Gillan, Mol. Phys. 38, 1781 (1979); G. M. Abernethy and M. J. Gillan, ibid. 39, 839 (1980).

${ }^{24}$ D. Beeman, J. Comput. Phys. 20, 130 (1976).

${ }^{25}$ P. I. C. Teixeira, J. M. Tavares, and M. M. Telo da Gama, J. Phys.: Condens. Matter 12, R411 (2000).

${ }^{26}$ H. Reiss, H. L. Frisch, and J. L. Lebowitz, J. Chem. Phys. 31, 369 (1959). 